medgen 2018 $\cdot 30: 391-399$

https://doi.org/10.1007/s11825-018-0217-z

Online publiziert:22. November 2018

(c) Der/die Autor(en) 2018

CrossMark
Florian Erger

Institut für Humangenetik, Uniklinik Köln, Köln, Deutschland

\title{
Monogene Formen der arteriellen Hypertonie
}

\section{Einleitung}

Die arterielle Hypertonie ist eine hochprävalente Erkrankung und betrifft in der deutschen Bevölkerung etwa jeden dritten Menschen [23]. Neben einem erhöhten Risiko für kardiovaskuläre Ereignisse stellt Bluthochdruck weltweit auch den zweithäufigsten Grund einer dialysepflichtigen Niereninsuffizienz dar [8]. Begünstigende Einflussfaktoren der primären oder essentiellen Hypertonie (ca. $90 \%$ aller Hypertoniker) sind unter anderem erhöhtes Alter, Nikotin- und Alkoholkonsum, Übergewicht, familiäre Belastung und Diabetes [4]. Genomweite Assoziationsstudien mit mehreren Hunderttausend Patienten haben in den letzten Jahren zahlreiche genetische Einflussfaktoren der essentiellen Hypertonie aufdecken können, die aber in der Summe nach jetzigem Kenntnisstand nur wenige Prozent der beobachteten Variabilität erklären [34]. Die erblichen Komponenten der essentiellen Hypertonie sind noch nicht vollständig aufgeklärt und deshalb auch im Rahmen der genetischen Routinediagnostik nicht in klinisch sinnvollem oder therapierelevantem Maße untersuchbar. Die genetischen Hintergründe der essentiellen Hypertonie werden in diesem Übersichtsartikel nicht weiter im Detail behandelt.

Etwa $10 \%$ der hypertensiven Patienten haben eine sekundäre Hypertonieform [26]. Der Anteil sekundärer Hypertonien ist unter jüngeren Patienten nochmals deutlich höher. Von Betroffenen <20 Jahren leidet die Mehrheit (50-60\%) an einer sekundären Hypertonie[12]. Zu dieser Krankheitsgruppe gehören beispielsweise Nierenarterienstenosen, Schlaf-Apnoe-Syndrom, primärer
Hyperaldosteronismus, Phäochromozytome, Hyperthyreose oder Hyperkortisolismus. Gerade bei sekundären Hypertonieformen ist der potenzielle therapeutische Nutzen einer gezielten Intervention oder medikamentösen Behandlung enorm.

Monogene Formen der arteriellen Hypertonie („hereditäre Hypertonien“ im engeren Sinne) werden pathophysiologisch den sekundären Hypertonien zugeordnet. Obwohl keine systematischen Studien zur kumulativen Häufigkeit von hereditären Hypertonien in der Gesamtbevölkerung existieren, dürfte ihr Anteil an sekundären Hypertonien in der Größenordnung von 1-2\% liegen [1, 14]. Der Anteil hereditärer Hypertonien am Gesamtkollektiv hypertensiver Patienten liegt dementsprechend in der Größenordnung von 1:1000, ist aber höher unter jüngeren Patienten.

\section{Hintergrund}

Die Blutdruckregulation durch die Nieren findet maßgeblich über die Aktivierung des Renin-Angiotensin-Aldosteron-Systems (RAAS) statt. Die Zellen des juxtaglomerulären Apparats des $\mathrm{Ne}$ phrons sezernieren das Enzym Renin u. a. als Reaktion auf eine abfallende glomeruläre Filtrationsrate (GFR), durch direkte $\beta$-adrenerge Stimulation, sowie als Reaktion auf einen niedrigen arteriellen Blutdruck. Renin führt im Blut als geschwindigkeitslimitierender Faktor zusammen mit dem Angiotensin-Converting Enzyme (ACE) über mehrere Zwischenschritte zur Aktivierung von Angiotensin II (AT-II). AT-II wiederum induziert in der Zona glomerulosa der Nebennierenrinde die Bildung und Ausschüttung des Mineralkortikoidhormons Aldosteron, wel- ches vielseitige blutdrucksteigernde Effekte hat. Hyperkaliämie ist ein weiterer potenter Reiz für die Aldosteronsekretion. In den Nieren führt Aldosteron nun über die Hochregulation der Expression des Natrium-Chlorid-Kotransporters (NCC) und des epithelialen Natriumkanals $(\mathrm{ENaC})$ in der apikalen Zellmembran der distalen Tubulus- und Sammelrohrzellen zu einer Natrium- und Flüssigkeitsrückresorption. Die Wiederaufnahme der positiv geladenen $\mathrm{Na}^{+}$-Ionen aus dem Lumen des Sammelrohres treibt über den so entstehenden elektrochemischen Gradienten die Sekretion von $\mathrm{K}^{+}$und $\mathrm{H}^{+}$-Ionen an; diese Ionen gehen dem Körper unter Aldosteroneinfluss also vermehrt verloren.

Nahezu alle bekannten hereditären Hypertonien betreffen pathomechanistisch direkt oder indirekt diese Regelkreise der renalen Elektrolyt- und/ oder Flüssigkeitshomöostase. Der hypertensive Effekt entsteht bei diesen Erkrankungen auf zweierlei Weise:

A. Durch eine erhöhte mineralkorti-

koide Wirkung, z. B. vermehrte

Aldosteronausschüttung (familiärer Hyperaldosteronismus Typ $I-I V$, PASNA-Syndrom [„,primary aldosteronism, seizures and neurologic abnormalities“, PASNA]), Synthese von Steroidvorstufen mit mineralkortikoidem Effekt (17 $\alpha$ - und $11 \beta$ Hydroxylasemangel) oder Verlust der Aldosteronspezifität des renalen $\mathrm{Mi}$ neralkortikoidrezeptors (apparenter Mineralkortikoidexzess, Hypertonus mit Exazerbation in der Schwangerschaft).

B. Durch eine pathologisch erhöhte Aktivität rückresorbierender Ionenkanäle des renotubulären Systems, z. B. durch reduzierten intrazellulären 


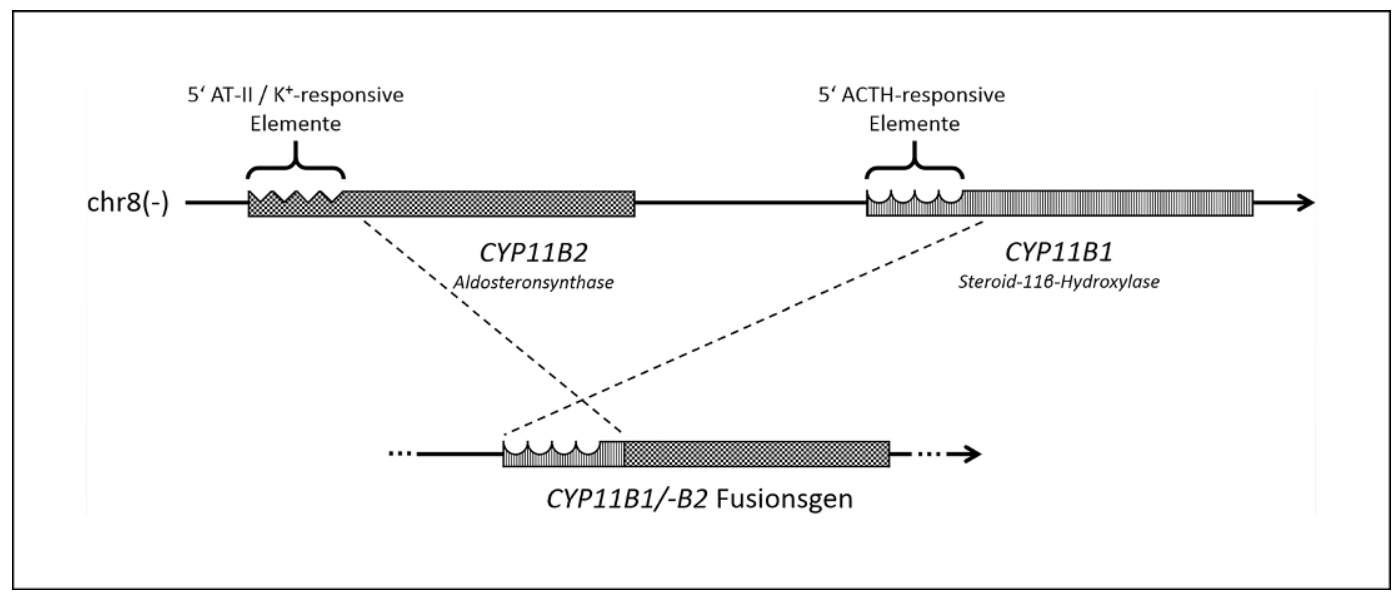

Abb. 1 A Ungleiches "crossing-over“ zwischen den zwei Genen CYP11B1 und CYP11B2 führt zu einem Fusionsgen, bei dem ACTH-responsive $5^{\prime}$-Elemente an die Aldosteronsynthasedomäne gekoppelt sind. CYP11B1 und CYP11B2 liegen benachbart auf dem (-)-Strang des Chromosoms 8.

AT-II Angiotensin II, ACTH adrenokortikotropes Hormon

Abbau (Liddle-Syndrom), erhöhte Expression an der Zelloberfläche (Pseudohypoaldosteronismus Typ II) oder erhöhte Offenwahrscheinlichkeit der Kanäle (auch LiddleSyndrom).

Alle genannten Formen gehen mit einer supprimierten Plasma-Renin-Aktivität einher. Die gemeinsame Endstrecke dieser Dysregulationen ist eine intravasale Volumenexpansion und arterielle Hypertonie.

\section{A. Erhöhte mineralkortikoide Wirkung}

\section{A1. Vermehrte \\ Aldosteronausschüttung}

Ein primärer Hyperaldosteronismus $(\mathrm{PH})$ als Ursache einer sekundären Hypertonie entsteht zum Beispiel infolge einer idiopathischen bilateralen Nebennierenrindenhyperplasie oder eines sporadischen Aldosteron produzierenden Adenoms. Es gibt jedoch auch z.T. deutliche familiäre Häufungen von Betroffenen mit $\mathrm{PH}$, die an einer monogenen Form des Hyperaldosteronismus leiden. Insgesamt werden fünf Unterarten des familiären Hyperaldosteronismus unterschieden, die allesamt einem autosomal-dominanten Erbgang folgen. Biochemisch wird ein PH durch einen erhöhten Quotienten aus Aldos- teronkonzentration und Plasma-ReninAktivität („aldosterone-to-renin ratio“, ARR) im Blut diagnostiziert.

\section{A1.1 Familiärer Hyperaldosteronismus Typ I}

Der familiäre Hyperaldosteronismus Typ I (FH1, OMIM \#103900) ist die erste und zugleich häufigste hereditäre Hypertonie, deren genetische Basis bisher aufgeklärt werden konnte [17].

Die Expression des CYP11B1-Gens (Steroid-11 $\beta$-Hydroxylase) in der Zona fasciculata der Nebennierenrinde führt zur Synthese von Glukokortikoiden und steht physiologischerweise unter der Kontrolle des adrenokortikotropen Hormons (ACTH) der Adenohypophyse. Die Synthese von Aldosteron geschieht hingegen in der Zona glomerulosa durch Expression von CYP11B2 (Aldosteronsynthase) und wird durch die Wirkung von Angiotensin II oder durch Hyperkaliämie induziert.

Die Ursache des FH1 ist ein chimäres Fusionsgen, bestehend aus Teilen der auf Chromosom 8 benachbart liegenden Gene CYP11B1 und CYP11B2. Durch ungleiches "crossing-over" und Fusion der ACTH-responsiven $5^{\prime}$-Regionen des CYP11B1-Gens mit der Aldosteronsynthasedomäne des CYP11B2-Gens kommt es zur pathologischen, ACTH-abhängigen und AT-II-entkoppelten Produktion von Aldosteron in der adrenalen Zona fasciculata (• Abb. 1). Die sehr hohe
Homologie dieser beiden Gene zueinander begünstigt dieses ungleiche „crossing-over". Berichte von unterschiedlichen Familien mit jeweils verschiedenen Rekombinationen belegen, dass die Mutation mehrfach unabhängig neu entstanden ist $[6,17]$.

Die Erstmanifestation liegt im Kindes- bis Jugendlichenalter. Patienten mit dieser Form der Hypertonie können zusätzlich eine Hypokaliämie und Alkalose aufweisen, diese Begleitsymptome sind aber fakultativ und fehlen bei milderen Fällen [6]. Die Expressivität ist auch intrafamiliär variabel. Es wurde eine erhöhte Inzidenz von intrakraniellen Blutungen und hämorrhagischen Insulten bei FH1 berichtet [18]. Alle hypertensiven Patienten mit primärem Hyperaldosteronismus $<20$ Jahre sollten auf FH1 untersucht werden [10]. Die genetische Diagnose ist per Long-Range-PCR unkompliziert und schnell möglich [15].

Die kausale Therapie des FH1 ist eine niedrig dosierte Glukokortikoidsubstitution (z. B. Dexamethason), um die hypophysäre ACTH-Sekretion zu unterdrücken. Mit Wegfall des ACTH-Stimulus sistiert die unphysiologische Aldosteronsynthese. Aus diesem Grund wird diese Hypertonieform auch „Glukokortikoid-supprimierbarer Hyperaldosteronismus" genannt. Um Glukokortikoide einzusparen, kann der Therapie Spironolacton hinzugefügt werden. 


\section{A1.2 Familiärer Hyperaldosteronismus Typ II}

Der familiäre Hyperaldosteronismus Typ II (FH2, OMIM \#605635) ist rein klinisch nicht vom FH1 unterscheidbar, lässt sich aber nicht durch Glukokortikoidsubstitution therapieren. In der Familie, in der $\mathrm{FH} 2$ erstmals klinisch beschrieben wurde [33], konnte jüngst CLCN2 als ursächliches Gen identifiziert werden [30]. Es ist noch unklar, welcher Anteil der vormals als $\mathrm{FH} 2$ diagnostizierten Patienten tatsächlich durch Mutationen in CLCN2 erklärt ist. CLCN2 kodiert ClC2, einen spannungsabhängigen Chloridkanal in den Aldosteron produzierenden Zellen der Nebennierenrinde. Die bisher identifizierten heterozygoten Mutationen in CLCN2 sind Gain-of-functionMutationen und führen zu einer erhöhten Offenwahrscheinlichkeit des ClC-2-Kanals [30]. Mit Öffnung von ClC-2 kommt es durch Chloridausstrom zur Membrandepolarisation und mittelbar zur Initiierung der Aldosteronsynthese (• Abb. 2). Eine inadäquat hohe Aldosteronproduktion ist die Folge. In der Therapie des FH2 sind Aldosteronantagonisten effektiv.

\section{A1.3 Familiärer Hyperaldosteronismus Typ III}

Typ III des familiären Hyperaldosteronismus (FH3, OMIM \#613677) wird durch konstitutionelle heterozygote Gain-offunction-Mutationen in KCNJ5 verursacht [5]. Rekurrente somatische Gainof-function-Mutationen in KCNJ5 finden sich auch in ca. $40 \%$ aller sporadischen Aldosteron produzierenden Adenome. Folge dieser Mutationen ist ein Selektivitätsverlust des von KCNJ5 kodierten und in der Zellmembran Aldosteron produzierender Zellen exprimierten Kaliumkanals GIRK4. Eine erhöhte Permeabilität des mutierten Kanals für $\mathrm{Na}^{+}$-Ionen bewirkt eine Membrandepolarisation, die wiederum über Zwischenschritte die Aldosteronsynthese aktiviert (• Abb. 2).

Der klinische Phänotyp des FH3 ist meist deutlich schwerer als bei anderen Formen des familiären Hyperaldosteronismus[21]. Patienten fallen in der Regel im Kleinkindesalter mit Hypokaliämie, Alkalose und schwerer arterieller Hypertonie auf. Bei der Mehrzahl der Patienten findet sich eine bilaterale Nebennieren-

medgen 2018· 30:391-399 https://doi.org/10.1007/s11825-018-0217-z

(c) Der/die Autor(en) 2018

\section{F. Erger}

\section{Monogene Formen der arteriellen Hypertonie}

\section{Zusammenfassung}

Hintergrund. Die Grundlage der arteriellen Hypertonie bei der überwiegenden Mehrzahl der Patienten ist multifaktorieller, zum großen Teil umweltbedingter Genese und derzeit in der Routinediagnostik nicht sinnvoll molekulargenetisch untersuchbar. Bei einem kleinen Teil der Patienten $(<1 \%)$ ist die arterielle Hypertonie aber Folge hereditärer, monogener Defekte. Hierzu gehören das Liddle-Syndrom, familiärer Hyperaldosteronismus, apparenter Mineralkortikoidexzess und Pseudohypoaldosteronismus. Diese und andere hereditäre Hypertonien gehen pathomechanistisch auf Störungen in den renalen Regelkreisen der Elektrolyt- und Volumenhomöostase zurück.

Ziel der Arbeit. Die bekannten Typen der hereditären Hypertonien sollen verständlich und schematisch erläutert werden. Besonderer Fokus liegt dabei auch auf dem klinischen
Bild der jeweiligen Unterformen sowie der therapeutischen Konsequenz, die sich in dieser Krankheitsgruppe direkt aus dem molekulargenetischen Befund ergeben kann. Eine Auswahl weiterer genetischer Syndrome mit arterieller Hypertonie als Begleit- oder Spätsymptom wird zusammenfassend behandelt.

Fazit. Die korrekte Diagnose eines Betroffenen mit einer Form der hier beschriebenen hereditären Hypertonien ermöglicht eine gezielte und effektive medikamentöse Therapie auf Basis der inzwischen insgesamt gut verstandenen jeweiligen Pathomechanismen.

\section{Schlüsselwörter}

Hereditäre Hypertonie - Liddle-Syndrom . Pseudohypoaldosteronismus · Familiärer Hyperaldosteronismus · AME

\section{Monogenic forms of arterial hypertension}

\section{Abstract}

Background. The cause of arterial hypertension in most patients is multifactorial, largely influenced by environmental factors, and currently not testable in routine molecular genetic diagnostics to a clinically useful degree. In a small subset of patients $(<1 \%)$ however, arterial hypertension is a consequence of hereditary monogenic defects. These include Liddle syndrome, familial hyperaldosteronism, apparent mineralocorticoid excess, and pseudohypoaldosteronism. Pathomechanistically, these and other hereditary forms of hypertension can be traced back to disturbances in the renal regulatory systems of electrolyte and volume homeostasis.

Objectives. The currently known types of hereditary hypertension are explained in a simplified, mechanistically oriented way.
This review will also focus on the clinical presentation of the forms of hereditary hypertension discussed and the therapeutic considerations that may arise directly from the molecular genetic testing results. A selection of additional genetic syndromes in which arterial hypertension is a secondary manifestation will be summarized. Conclusions. The correct diagnosis of a patient with a form of the hereditary hypertension described here enables a targeted and effective pharmacological therapy based on the now generally wellunderstood pathomechanisms.

\section{Keywords}

Hereditary hypertension - Liddle syndrome . Pseudohypoaldosteronism · Familial hyperaldosteronism · AME hyperplasie. Ein Therapieversuch mit Aldosteronantagonisten sollte unternommen werden - schwere Fälle zeigen sich jedoch z.T. hierauf resistent und benötigen eine bilaterale Adrenalektomie zur adäquaten Kontrolle ihres Blutdrucks.

\section{A1.4 Familiärer Hyperaldosteronismus Typ IV}

Eine weitere Form, der familiäre Hyperaldosteronismus Typ IV (FH4, OMIM \#617027), wurde 2015 bei fünf nicht verwandten Patienten in einem Kollektiv von Kindern mit frühmanifestem (<10 Jahre) Hyperaldosteronismus erstmals beschrieben [29]. Ursächlich sind 


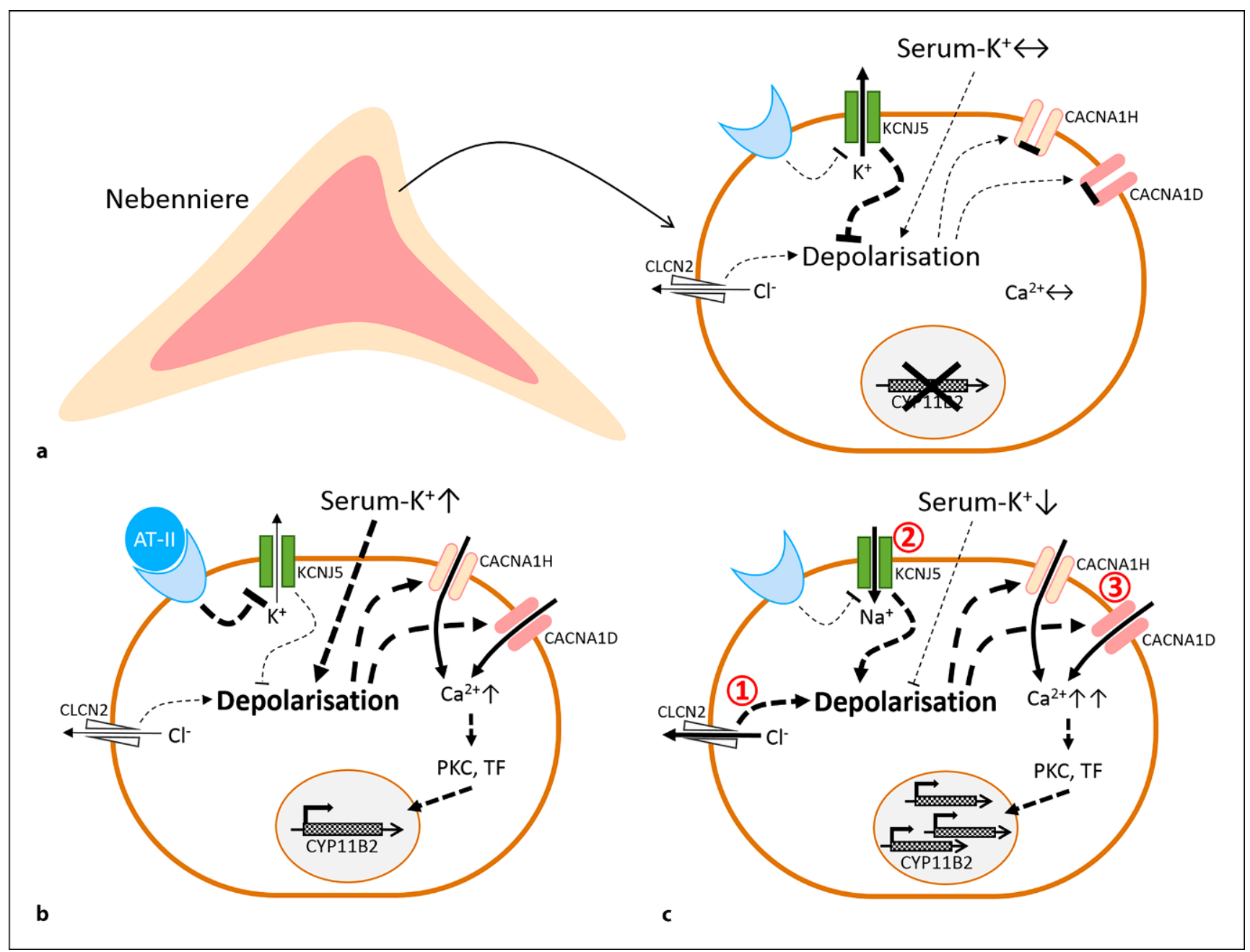

Abb. 2 ム Aldosteron produzierende Zelle der Zona glomerulosa der Nebennierenrinde.

a Inaktivität: Die Membranhyperpolarisation wird durch KCNJ5 aufrechterhalten, es findet keine Aldosteronproduktion statt. b Physiologische Aktivierung: Durch Bindung von AT-Il und konsekutiver Hemmung des KCNJ5 oder durch Hyperkaliämie wird eine Membrandepolarisation ausgelöst. Spannungsabhängige Kalziumkanäle öffnen sich und $\mathrm{Ca}^{2+}$ strömt ein. Es wird eine Proteinkinase- und Transkriptionsfaktorkaskade ausgelöst, die die Expression der Aldosteronsynthase induziert. c Pathologische Aktivierung: Beim FH2 ist CLCN2 pathologisch geöffnet und führt zur Membrandepolarisation durch $\mathrm{Cl}^{-}$-Ausstrom (1). Beim FH3 verliert KCNJ5 seine lonenselektivität und $\mathrm{Na}+$ strömt ein, was wiederum zu einer Membrandepolarisation führt (2)). Bei FH4 und beim PASNA sind die Kalziumkanäle der Zelle auch ohne Depolarisationsreiz geöffnet und es kommt zum gesteigerten Kalziuminflux (3).

AT-II Angiotensin II, FH familiärer Hyperaldosteronismus, PASNA „primary aldosteronism, seizures and neurologic abnormalities", PKC Ca ${ }^{2+}$-abhängige Proteinkinasen, TF Transkriptionsfaktoren

heterozygote Gain-of-function-Mutationen im Gen $C A C N A 1 H$, welches für einen T-Typ-Kalziumkanal $(\mathrm{T}=$ transient $)$ in Aldosteron produzierenden Zellen kodiert. Die bisher detektierten Mutationen führen zu einer deutlich verlängerten Inaktivierungszeit der Kanäle und zu einem erhöhten Kalziuminflux in die Zelle, der eine inadäquat gesteigerte $\mathrm{Al}-$ dosteronproduktion bewirkt ( $\mathbf{A} \mathbf{A b b}$. 2). Patienten mit FH4 werden im Kindesbis Jugendlichenalter symptomatisch. Es besteht in der Regel keine makro- skopische Nebennierenhyperplasie. Die Penetranz des FH4 ist vermutlich hoch, aber nicht vollständig [29]. Aldosteronantagonisten sind die Mittel der Wahl, allerdings ist aufgrund der Seltenheit der Erkrankung derzeit noch nicht klar, bei welchem Anteil der Patienten diese medikamentöse Monotherapie ausreichend ist. Die Wirksamkeit von Kalziumkanalblockern bei Patienten mit FH4 ist noch unzureichend untersucht.

\section{A1.5 PASNA-Syndrom}

Eine ähnliche Form der hereditären Hypertonie ist das sog. PASNA-Syndrom (OMIM \#615474). Hierbei ist das Gen CACNA1D, welches einen L-Typ-Kalziumkanal ( $\mathrm{L}=$ langandauernd) kodiert, von heterozygoten Gain-of-functionMutationen betroffen [28]. Einzelne Patienten sind beschrieben. Der Pathomechanismus hinsichtlich der Hypertonie entspricht weitgehend dem des FH4, jedoch treten zusätzliche extraadrenale Manifestationen, insbesondere Epilep- 


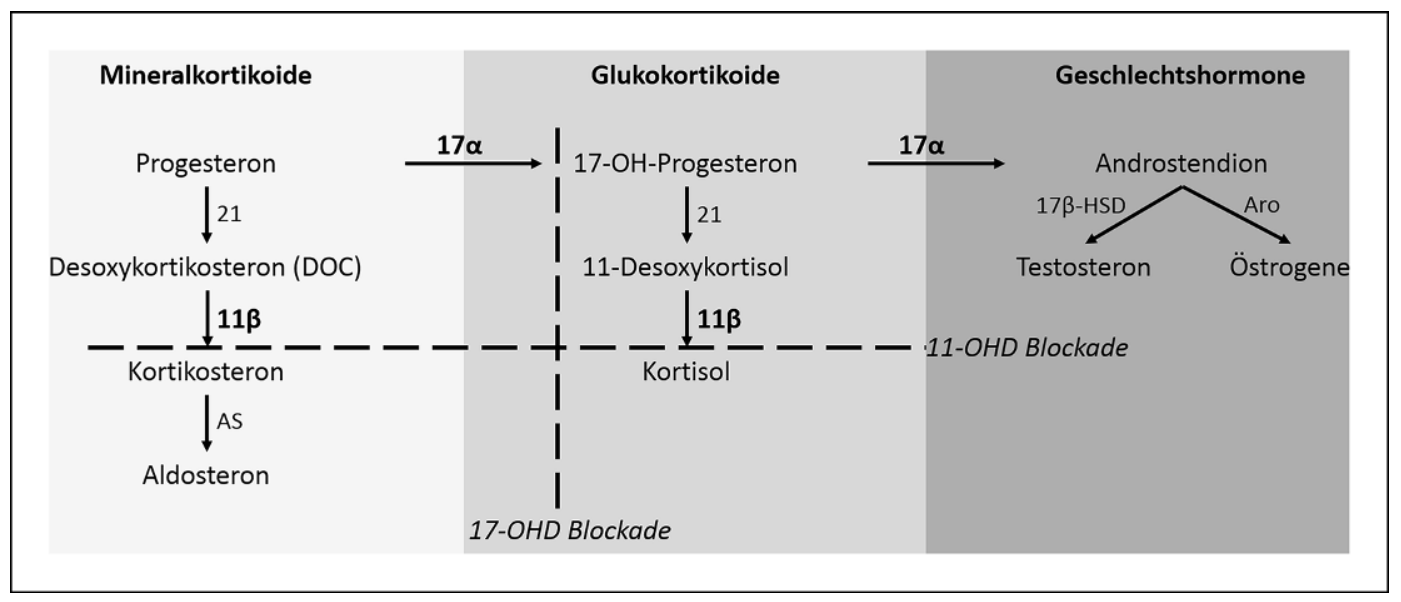

Abb. 3 ॥ Steroidhormonsynthese, ausgehend von Progesteron. Vorstufen proximal des jeweiligen Enzymblocks werden unter ACTH-Stimulus vermehrt produziert.

21 21-Hydroxylase, 17a 17a-Hydroxylase, $11 \beta 11 \beta$-Hydroxylase, 11-OHD 11 $\beta$ Hydroxylasemangel, 17-OHD 17a Hydroxylasemangel, 17 $\beta$-HSD 17 $\beta$-Hydroxysteroiddehydrogenase, Aro Aromatase, AS Aldosteronsynthase

sie, spastische Bewegungsstörungen und Intelligenzminderung auf. Auch wurden Gain-of-function-Mutationen in CACNA1D mit Autismus bzw. hyperinsulinämer Hypoglykämie assoziiert [7, 25]. Als Grund für das breitere phänotypische Spektrum wird die stärkere zerebrale Expression und insgesamt höhere Konduktivität des L-Typ-Kalziumkanals gegenüber dem T-Typ-Kalziumkanal angenommen. Ein Patient mit PASNASyndrom zeigte ein sehr gutes Ansprechen des Blutdrucks auf eine Therapie mit dem Kalziumkanalblocker Amlodipin [28].

\section{A2. Synthese von Steroidvorstufen mit mineralkortikoidem Effekt}

In dieser Gruppe sind der 17a-Hydroxylasemangel (17-OHD, OMIM \#202110) und der 11ß-Hydroxylasemangel (11-OHD, OMIM \#202010) von Bedeutung. Bei beiden handelt es sich um seltene Subtypen des autosomalrezessiven adrenogenitalen Syndroms (AGS). Der 11-OHD macht etwa 5\% aller AGS aus, der 17-OHD ist nochmals deutlich seltener $(<1 \%$ aller AGS) [24]. Beiden Formen ist eine mangelnde Kortisolsynthese aufgrund der jeweiligen Enzymdefizienz gemeinsam. Unter reaktiv erhöhter ACTH-Stimulation produzieren die Nebennieren vermehrt Steroidvorstufen wie das Desoxykortikosteron (DOC), die eine mineralkorti- koide Wirkung haben (• Abb. 3) und diagnostisch im Blut bestimmt werden können.

Sowohl der 11-OHD als auch der 17-OHD führen zu Hypertonie, Alkalose und Hypokaliämie, abhängig von der jeweiligen enzymatischen Restfunktion. Patienten mit 17-OHD weisen zudem eine reduzierte Synthese von Geschlechtshormonen auf. Bei weiblichen Betroffenen äußert sich dies in einem hypergonadotropen Hypogonadismus mit Amenorrhoe und Pubertas tarda, bei chromosomal männlichen Betroffenen mit intersexuellem oder phänotypisch weiblichem äußeren Genitale bei Geburt. Beim 11-OHD kommt es hingegen spiegelbildlich zur erhöhten Produktion von Geschlechtshormonen: Weibliche Betroffene zeigen eine Virilisierung bis hin zu intersexuellem Genitale, männliche Betroffene entwickeln eine Pubertas praecox. Eine medikamentöse Glukokortikoidsubstitution (und Geschlechtshormonsubstitution im Falle der 17OHD) korrigiert den endokrinologischen Phänotyp durch Supprimierung der pathologischen ACTH-Stimulation.

\section{A3. Spezifitätsverlust des renalen Mineralkortikoidrezeptors}

\section{A3.1 Syndrom des apparenten Mineralkortikoidexzesses}

Der renale Mineralkortikoidrezeptor (MR) lässt sich in vitro sowohl durch
Aldosteron als auch durch Kortisol aktivieren [9]. In vivo findet jedoch eine Aktivierung des renalen MR durch Kortisol dank des Enzyms 11 $\beta$-Hydroxysteroiddehydrogenase Typ II (HSD11B2) nicht statt. Aufgabe dieses renal und gastrointestinal exprimierten Enzyms ist der Umsatz des bioaktiven Kortisols in sein inaktives Metabolit Kortison, bevor es zur Bindung von Kortisol an den MR kommen kann. Bei dem autosomal-rezessiven AME (apparenter Mineralkortikoidexzess; OMIM \#218030) ist die Enzymfunktion der HSD11B2 durch Loss-of-function-Mutationen im gleichnamigen Gen reduziert, sodass die HSD11B2 vermittelte Aldosteronselektivität des MR verloren geht. Es resultiert eine unphysiologische Aktivierung des renalen MR durch Kortisol. Diagnostisch wegweisend ist ein hoher Kortisol/ Kortison-Quotient im Urin.

AME tritt bereits im Kleinkindesalter auf und geht mit z.T. schwerer Alkalose und Hypokaliämie einher. Zusätzlich besteht eine Hyperkalziurie mit Neigung zur Nephrolithiasis [20]. Aldosteronantagonisten, optimalerweise in Kombination mit Salzrestriktion, sind wirksam.

\section{A3.2 Hypertonus mit Exazerbation in der Schwangerschaft}

Für diese äußerst seltene autosomal-dominante hereditäre Hypertonie (OMIM \#605115) wurde bis dato nur eine Familie mit 12 Betroffenen beschrieben[11]. 


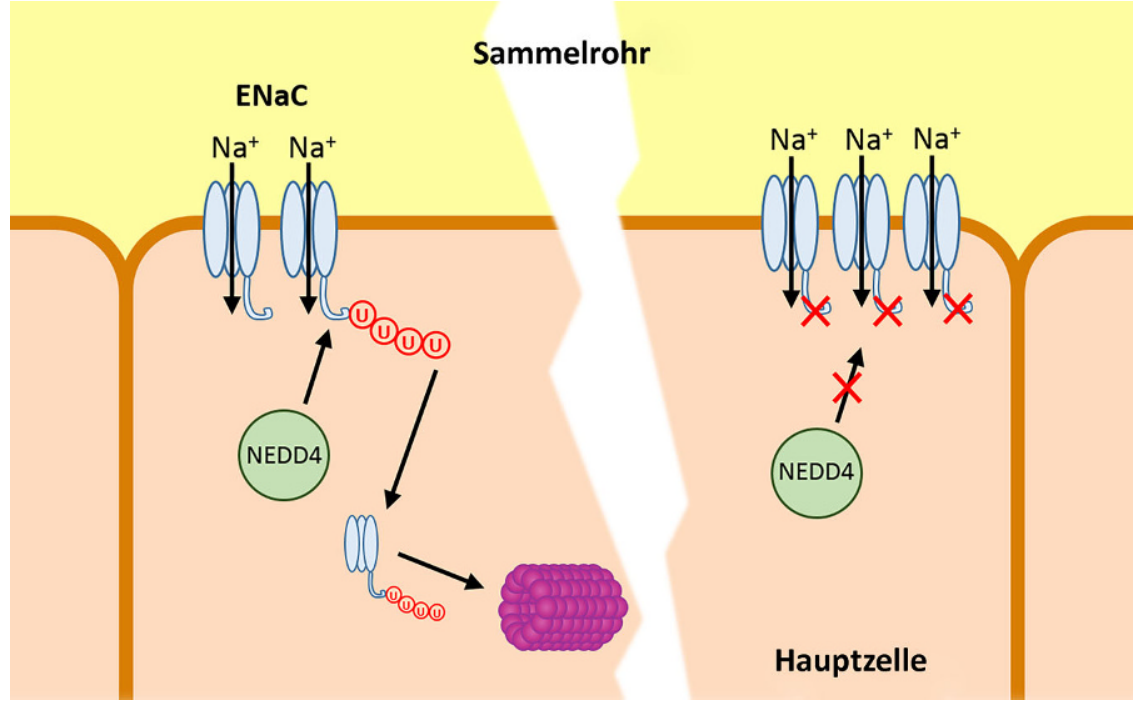

Abb. 4 ॥ Pathomechanismus des Liddle-Syndroms in den Hauptzellen des Sammelrohrs. Links: physiologische Situation. Rechts: Durch Mutationen im C-terminalen zytoplasmatischen Teil der ENaC-Untereinheiten $\beta$ und $\gamma$ wird eine Ubiquitinylierung durch die E3 Ubiquitin-Proteinligase NEDD4 verhindert. Der Kanal verbleibt in der apikalen Zellmembran. Mutationen in der a-Untereinheit erhöhen die Offenwahrscheinlichkeit des $\mathrm{ENaC}$ (nicht dargestellt).

ENaC epithelialer Natriumkanal

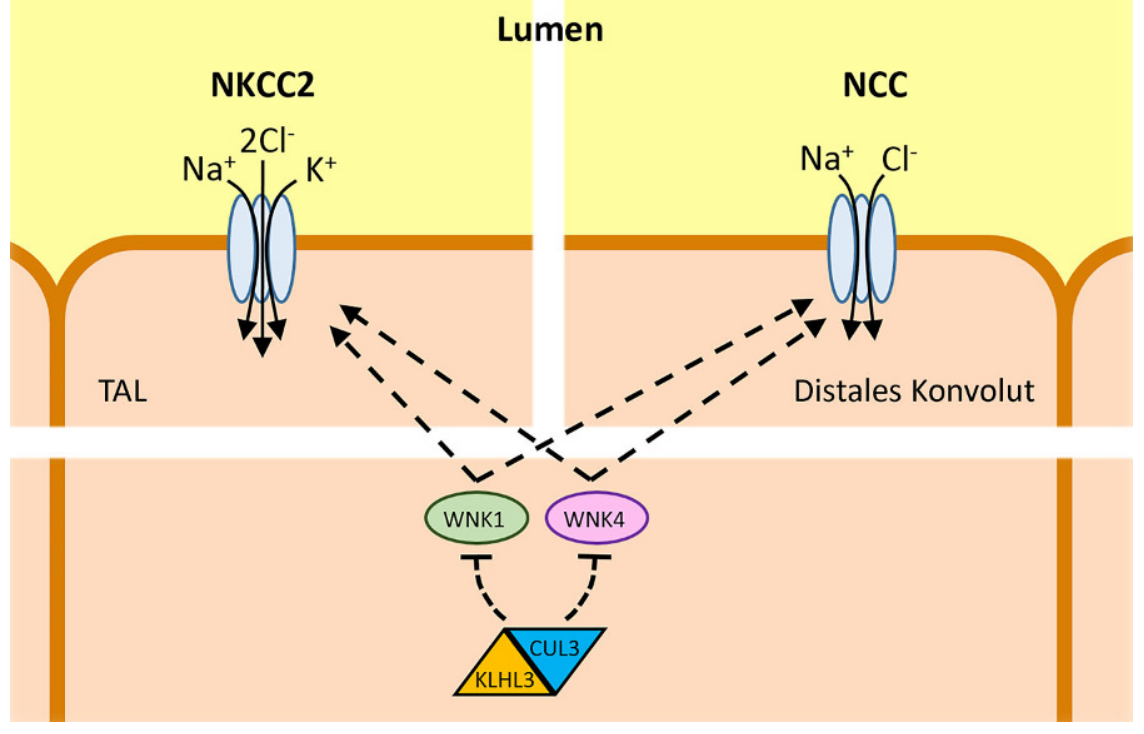

Abb. 5 A Regulation des Natrium-Kalium-2Chlorid-Kotransporters (NKCC2) im aufsteigenden Schenkel der Henle'schen Schleife (TAL) und des Natrium-Chlorid-Kotransporters (NCC) im distalen Konvolut durch WNK1 und WNK4. KLHL3 und CUL3 hemmen die WNK-Kinase-Aktivität durch Induktion des proteasomalen Abbaus

Ursächlich ist die Gain-of-function-Mutation Ser810Leu des Mineralkortikoidrezeptor-Gens NR3C2. Diese Mutation führt zu einer permanenten mittelgradigen Aktivierung des MR sowie zu einem Spezifitätsverlust der Ligandenbindungsstelle. Sowohl Progesteron wie auch andere Steroide aktivieren den mutierten MR.
Spironolacton/Eplerenon sind kontraindiziert, da auch sie einen aktivierenden Effekt auf den mutierten MR haben. Die bisher beschriebenen Betroffenen wurden mit Thiaziden, kaliumsparenden Diuretika und Salzrestriktion behandelt. Bei Schwangeren mit unkontrollierbarer Exazerbation des Hypertonus ist die Entbindung des Feten die einzige Behandlungsoption. Neuere, derzeit in Phase-III-Studien befindliche MR-Blocker wirken auch antagonistisch auf den Ser810Leu-MR [2] und könnten sich möglicherweise zur antihypertensiven Therapie dieser Patienten eignen.

\section{B. Erhöhte Aktivität renotubulärer Ionenkanäle}

\section{B1. Liddle-Syndrom}

Das autosomal-dominante Liddle-Syndrom (OMIM \#177200) wurde erstmals 1963 in einer Familie mit frühmanifester Hypertonie und Hypokaliämie beschrieben [16]. Als genetische Grundlage wurden seit 1994 bis heute mehrere Gain-of-function-Mutationen in den Genen SCNN1A, SCNN1B und SCNN1G - entsprechend den drei Untereinheiten des epithelialen Natriumkanals ( $\mathrm{ENaC})$ in den Hauptzellen des Sammelrohres - identifiziert $[13,27,32]$. Die beschriebenen Mutationen in SCNN1B und SCNN1G liegen am intrazellulären C-Terminus des kodierten Proteins und zerstören ein Bindungsmotiv, über das $\mathrm{ENaC}$ dem proteasomalen Abbau zugeführt wird. Die Anzahl membranständiger $\mathrm{ENaC}$ steigt und die $\mathrm{Na}^{+}-$ Resorption (sekundär auch die Wasserrückaufnahme) im Sammelrohr nimmt $\mathrm{zu}$ (•Abb.4). Die einzige bisher berichtete Liddle assoziierte Mutation in SCNN1A (Cys479Arg) betrifft den extrazellulären Teil des $\mathrm{ENaC}$ und erhöht die Offenwahrscheinlichkeit des Kanals.

Der Phänotyp des Liddle-Syndroms ist ein hyporeninämer, hypoaldosteronämer Bluthochdruck im Kindes- bis jungen Erwachsenenalter. Alkalose und Hypokaliämie sind meist milde ausgeprägt. $\mathrm{Da}$ die erhöhte $\mathrm{Na}^{+}$-Rückresorption nicht Aldosteron vermittelt ist, sind Aldosteronantagonisten nicht wirksam. Die kaliumsparenden Diuretika Ami- 
Tab. 1 Ausgewählte weitere genetische Syndrome mit arterieller Hypertonie als Begleit- oder Spätsymptom

\begin{tabular}{|c|c|c|c|c|}
\hline Syndrom & Gen & Erbgang & Phänotyp & Ursache des Hypertonus \\
\hline $\begin{array}{l}\text { Hypertonus-Brachydaktylie- } \\
\text { Syndrom }\end{array}$ & PDE3A & $A D$ & $\begin{array}{l}\text { Verkürzte Metakarpal- und Metatarsal- } \\
\text { knochen, verkürzte Phalangen, } \\
\text { milder proportionierter Kleinwuchs }\end{array}$ & $\begin{array}{l}\text { Proliferationsreiz } \rightarrow \text { generalisierte } \\
\text { Hyperplasie der Gefäßwände }\end{array}$ \\
\hline \multirow{5}{*}{$\begin{array}{l}\text { Phäochromozytom-, } \\
\text { Paragangliom-Syndrome }\end{array}$} & $S D H A$ & \multirow[t]{5}{*}{$A D$} & \multirow{5}{*}{$\begin{array}{l}\text { (Multiple) Phäochromozytome und/oder } \\
\text { Paragangliome, anfallsartige Tachykardien, } \\
\text { Schwitzen, Blässe }\end{array}$} & \multirow[t]{5}{*}{ Katecholamine } \\
\hline & $S D H B$ & & & \\
\hline & $S D H C$ & & & \\
\hline & $S D H D$ & & & \\
\hline & SDHAF2 & & & \\
\hline Neurofibromatose Typ I & NF1 & $A D$ & $\begin{array}{l}\text { Kutane Neurofibrome, Café-au-lait-Flecken, } \\
\text { Lisch-Knoten, Optikusgliom }\end{array}$ & $\begin{array}{l}\text { Nierenarterienstenose oder } \\
\text { Phäochromozytome }\end{array}$ \\
\hline Williams-Beuren-Syndrom & $\begin{array}{l}\text { Deletion } \\
7 q 11.23\end{array}$ & $A D$ & $\begin{array}{l}\text { Neonatale Hyperkalzämie, faziale } \\
\text { Dysmorphien, Herzfehler, Kleinwuchs, } \\
\text { Schwerhörigkeit }\end{array}$ & $\begin{array}{l}\text { Nierenarterienstenose, } \\
\text { kardiovaskuläre Fehlbildungen }\end{array}$ \\
\hline Grange-Syndrom & YY1AP1 & AR & $\begin{array}{l}\text { Multiple arterielle Stenosen, Herzfehler, } \\
\text { Brachydaktylie, Syndaktylie, Knochen- } \\
\text { brüchigkeit }\end{array}$ & Nierenarterienstenose \\
\hline $\begin{array}{l}\text { Syndrome mit signifikanter } \\
\text { Nephropathie }\end{array}$ & Diverse & - & $\begin{array}{l}\text { z. B. Nierenzysten (PKD), } \\
\text { Angiomyolipome (TSC) }\end{array}$ & Niereninsuffizienz \\
\hline
\end{tabular}

lorid und Triamteren blockieren ENaC direkt und sind die Mittel der Wahl in diesen Patienten. Der Behandlungserfolg ist in der Regel gut.

\section{B2. Pseudohypoaldosteronismus Typ II (PHA2)}

Der PHA2, auch Gordon-Syndrom genannt, hat derzeit fünf genetisch unterscheidbare Subtypen (PHA2A-E, OMIM PS145260), die autosomal-dominant erblich sind. Der Typ D ist zusätzlich auch autosomal-rezessiv erblich. Die Ursache des PHA2A ist nicht bekannt, Linkagestudien zeigen eine Assoziation zur chromosomalen Region 1q31-q42 [19]. PHA2B wird durch heterozygote Mutationen in WNK4, PHA2C durch heterozygote Mutationen in WNK1 ausgelöst. Sowohl in WNK4 als auch in WNK1 sind Gain-of-function-Mutationen ursächlich. Beide Gene kodieren für sog. WNK-Kinasen, die u. a. in den Tubuluszellen des distalen Konvoluts die apikale Expression des Thiazid sensitiven Natrium-Chlorid-Kotransporters (NCC) und im aufsteigenden Schenkel der Henle'schen Schleife den Natrium-Kalium2Chlorid-Kotransporter (NKCC2) positiv regulieren [35]. PHA2D entsteht durch mono- oder biallelische Loss-of-
function-Mutationen in KLHL3, PHA2E durch heterozygote Loss-of-functionMutationen in CUL3. KLHL3 und CUL3 kodieren für zwei Proteine, die beide Teil des sog. E3-Ligase-Komplexes sind, dessen Funktion die Ubiquitinylierung der WNK-Kinasen beinhaltet [31]. Eine reduzierte Aktivität dieses Komplexes führt also wiederum $\mathrm{zu}$ einer Funktionssteigerung der WNK-Kinasen mit pathologisch erhöhter apikaler Expression von NCC und NKCC2 (• Abb. 5). $\mathrm{Na}^{+}, \mathrm{Cl}^{-}$und $\mathrm{K}^{+}$werden vermehrt resorbiert, saure Valenzen in Form von $\mathrm{H}^{+}$aufgrund eines reduzierten elektrochemischen Gradienten im Sammelrohr weniger sezerniert.

Alle Subformen des PHA2 gehen typischerweise mit Hyperchlorämie, Hyperkaliämie und Azidose einher. Renin ist supprimiert, Aldosteron aber aufgrund des stimulatorischen Effekts der Hyperkaliämie meist normwertig oder erhöht. Patienten mit Mutationen in WNK1, WNK4 und KLHL3 haben eine eher spätere Manifestation im Jugendlichen- oder jungen Erwachsenenalter. Der CUL3-assoziierte PHA2E tritt im Kindesalter auf und führt im Mittel zu größeren metabolischen Abweichungen als die anderen Subtypen [3]. In der Therapie werden Thiazide zur Blockade des NCC eingesetzt und normalisieren die Elektrolytabweichungen und den Hypertonus bei fast allen Patienten.

Ähnliche Elektrolytabweichungen wie beim PHA2 bestehen auch beim Typ I des Pseudohypoaldosteronismus (PHA1). PHA1 geht aber nicht mit einer Hypertonie einher, sondern mit Neigung zur hyperreninämen Hypotonie und renalem Salzverlust. Ursächlich sind heterozygote pathogene Varianten in NR3C2 sowie biallelische pathogene Varianten in SCNN1A, SCNN1B oder $S C N N 1 G$ - hier allerdings im Gegensatz zum Liddle-Syndrom als Loss-offunction-Mutationen.

\section{Weitere Syndrome mit Hypertonus als Begleitsymptom}

Eine arterielle Hypertonie ist Begleitoder Spätsymptom in zahlreichen monogenen Syndromen. Eine Auswahl von besonders relevanten Erkrankungen ist in - Tab. 1 zusammengestellt.

\section{Resümee}

Hereditäre Hypertonien sind seltene genetische Erkrankungen, die - anders als die essentielle Hypertonie - vor allem junge Menschen betreffen. Die korrekte 


\begin{tabular}{|c|c|c|c|c|c|c|c|}
\hline Typ & Gen & Erbgang & Elektrolyte & Aldosteron & Therapie & $\begin{array}{l}\text { Relative } \\
\text { Häufigkeit* }\end{array}$ & Sonstiges \\
\hline FH1 & $C Y P 11 B 1 /-B 2$ & $A D ; G o F$ & $\begin{array}{l}\mathrm{K}^{+} \downarrow / \leftrightarrow \\
\mathrm{H}^{+} \downarrow / \leftrightarrow\end{array}$ & $\uparrow$ & Glukokortikoide & ++++ & $\begin{array}{l}\text { Intrakranielle } \\
\text { Blutungen }\end{array}$ \\
\hline $\mathrm{FH} 2$ & CLCN2 & $A D ; G o F$ & $\begin{array}{l}\mathrm{K}^{+} \downarrow / \leftrightarrow \\
\mathrm{H}^{+} \downarrow / \leftrightarrow\end{array}$ & $\uparrow$ & MRA & ++ & - \\
\hline $\mathrm{FH} 3$ & KCNJ5 & $A D ; G o F$ & $\begin{array}{l}\mathrm{K}^{+} \downarrow \\
\mathrm{H}^{+} \downarrow\end{array}$ & $\uparrow$ & MRA, bilat. Adrenalektomie & ++ & NN-Hyperplasie \\
\hline $\mathrm{FH} 4$ & CACNA1H & $A D ; G o F$ & $\begin{array}{l}\mathrm{K}^{+} \downarrow / \leftrightarrow \\
\mathrm{H}^{+} \downarrow / \leftrightarrow\end{array}$ & $\uparrow$ & MRA & ++ & - \\
\hline PASNA & CACNA1D & $A D ; G o F$ & $\begin{array}{l}\mathrm{K}^{+} \downarrow \\
\mathrm{H}^{+} \downarrow\end{array}$ & $\uparrow$ & MRA, (Ca ${ }^{2+}-$ Kanalblocker?) & + & Epilepsie, Spastik \\
\hline 11-OHD & CYP11B1 & AR; LoF & $\begin{array}{l}\mathrm{K}^{+} \downarrow / \leftrightarrow \\
\mathrm{H}^{+} \downarrow / \leftrightarrow\end{array}$ & $\downarrow$ & Glukokortikoide & ++++ & - \\
\hline 17-OHD & CYP17A1 & AR; LoF & $\begin{array}{l}\mathrm{K}^{+} \downarrow / \leftrightarrow \\
\mathrm{H}^{+} \downarrow / \leftrightarrow\end{array}$ & $\downarrow$ & $\begin{array}{l}\text { Glukokortikoide, } \\
\text { Geschlechtshormone }\end{array}$ & ++++ & Hypogonadismus \\
\hline AME & HSD11B2 & AR; LoF & $\begin{array}{l}\mathrm{K}^{+} \downarrow \\
\mathrm{H}^{+} \downarrow\end{array}$ & $\downarrow$ & MRA, Salzrestriktion & +++ & - \\
\hline HES & $N R 3 C 2$ & $A D ; G o F$ & $\begin{array}{l}\mathrm{K}^{+} \downarrow \\
\mathrm{H}^{+} \downarrow\end{array}$ & $\downarrow$ & $\begin{array}{l}\text { Thiazide, Amilorid, } \\
\text { Triamteren, Salzrestrikti- } \\
\text { on, SPL/EPL kontraindiziert }\end{array}$ & + & $\begin{array}{l}\text { Exazerbation in } \\
\text { Schwangerschaft }\end{array}$ \\
\hline \multirow[t]{3}{*}{ Liddle } & SCNN1A & $A D ; G o F$ & \multirow{3}{*}{$\begin{array}{l}\text { Alle Formen: } \\
\mathrm{K}^{+} \downarrow / \leftrightarrow \\
\mathrm{H}^{+} \downarrow / \leftrightarrow\end{array}$} & \multirow[t]{3}{*}{$\downarrow$} & \multirow[t]{3}{*}{ Amilorid, Triamteren } & \multirow[t]{3}{*}{+++} & - \\
\hline & SCNN1B & $A D ; G o F$ & & & & & - \\
\hline & SCNN1G & $A D ; G o F$ & & & & & - \\
\hline \multirow[t]{4}{*}{ PHA2 } & WNK4 & $A D ; G o F$ & \multirow{4}{*}{$\begin{array}{l}\text { Alle Formen: } \\
\mathrm{K}^{+} \uparrow \\
\mathrm{H}^{+} \uparrow \\
\mathrm{Cl}^{-} \uparrow\end{array}$} & \multirow[t]{4}{*}{$\leftrightarrow / \uparrow$} & \multirow[t]{4}{*}{ Thiazide } & \multirow[t]{4}{*}{ Kumulativ +++} & - \\
\hline & WNK1 & $A D ; G o F$ & & & & & - \\
\hline & $K L H L 3$ & $A D, A R ;$ LoF & & & & & - \\
\hline & CUL3 & $A D ;$ LoF & & & & & Schwerere Klinik \\
\hline \multicolumn{8}{|c|}{ 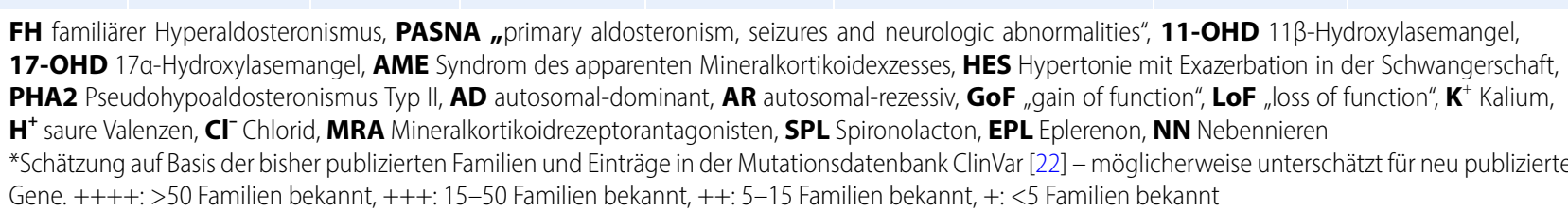 } \\
\hline
\end{tabular}

klinische und genetische Zuordnung $\mathrm{zu}$ einem der hier beschriebenen Syndrome hat für Patienten eine direkte, unmittelbare therapeutische Konsequenz, da sich die meisten hereditären Hypertonien kausal und effektiv behandeln lassen.

Die hier genannten Formen der monogenen arteriellen Hypertonie sind in - Tab. 2 nochmals zusammenfassend gegenübergestellt.

\section{Fazit für die Praxis}

- Hereditäre, monogene Hypertonien entstehen durch eine erhöhte Mineralkortikoidwirkung oder eine inadäquat gesteigerte Aktivität der Ionenkanäle des renalen Tubulussystems.
- Alle nichtsyndromalen Formen sind typischerweise hyporeninäm.

- Sie betreffen vor allem junge Erwachsene und Kinder.

- Bei dieser Patientengruppe sollte frühzeitig eine genetische Diagnostik erwogen werden, da sich daraus bei positivem Testergebnis eine direkte therapeutische Relevanz mit unmittelbarem Benefit für den Patienten ergibt.

- Konkret sollten auch alle hypertensiven Patienten mit primärem Hyperaldosteronismus unter 20 Jahren auf familiären Hyperaldosteronismus Typ I getestet werden.

- Elektrolytverschiebungen sind bei vielen Formen nicht obligat und ihr Fehlen kann in die Irre führen.
- Die Expressivität ist variabel - ein (noch) normaler Blutdruck bei einem Verwandten eines Betroffenen schließt die Diagnose i.d. R. nicht sicher aus.

\section{Korrespondenzadresse}

Dr. med. Florian Erger Institut für Humangenetik, Uniklinik Köln Kerpener Str. 34, 50931 Köln, Deutschland florian.erger@uk-koeln.de

\section{Einhaltung ethischer Richtlinien}

Interessenkonflikt. F. Erger gibt an, dass kein Interessenkonflikt besteht.

Dieser Beitrag beinhaltet keine von den Autoren durchgeführten Studien an Menschen oder Tieren. 
Open Access. Dieser Artikel wird unter der Creative Commons Namensnennung 4.0 International Lizenz (http://creativecommons.org/licenses/by/4.0/deed. de) veröffentlicht, welche die Nutzung, Vervielfältigung, Bearbeitung, Verbreitung und Wiedergabe in jeglichem Medium und Format erlaubt, sofern Sie den/die ursprünglichen Autor(en) und die Quelle ordnungsgemäßnennen, einen Link zur Creative Commons Lizenz beifügen und angeben, ob Änderungen vorgenommen wurden.

\section{Literatur}

1. Aglony M, Martinez-Aguayo A, Carvajal CA et al (2011) Frequency of familial hyperaldosteronism type 1 in a hypertensive pediatric population: clinical and biochemical presentation. Hypertension 57:1117-1121

2. Amazit L, Le Billan F, Kolkhof P et al (2015) Finerenone impedes Aldosterone-dependent nuclear import of the Mineralocorticoid receptor and prevents genomic recruitment of steroid receptor Coactivator-1. JBiol Chem 290:21876-21889

3. Boyden LM, Choi M, Choate KA et al (2012) Mutations in kelch-like 3 and cullin 3 cause hypertension and electrolyte abnormalities. Nature 482:98-102

4. Chalmers J, Macmahon S, Mancia G et al (1999) 1999 World Health Organization-International Society of Hypertension Guidelines for the management of hypertension. Guidelines subcommittee of the World Health Organization. Clin ExpHypertens 21:1009-1060

5. Choi M, Scholl UI, Yue P et al (2011) K+ channel mutations in adrenal aldosterone-producing adenomas and hereditary hypertension. Science 331:768-772

6. Dluhy RG, Lifton RP (1995) Glucocorticoidremediable aldosteronism (GRA): diagnosis, variability of phenotype and regulation of potassium homeostasis. Steroids 60:48-51

7. Flanagan SE, Vairo F, Johnson MB et al (2017) A CACNA1D mutation in a patient with persistent hyperinsulinaemic hypoglycaemia, heart defects, and severe hypotonia. Pediatr Diabetes 18:320-323

8. Foley RN, Collins AJ (2007) End-stage renal disease in the united states: an update from the united states renal data system. J Am Soc Nephrol 18:2644-2648

9. FreyFJ, Odermatt A, Frey BM (2004) Glucocorticoidmediated mineralocorticoid receptor activation and hypertension. Curr Opin Nephrol Hypertens 13:451-458

10. Funder JW, Carey RM, Mantero F et al (2016) The management of primary aldosteronism: case detection, diagnosis, and treatment: an endocrine society clinical practice guideline. J Clin Endocrinol Metab 101:1889-1916

11. Geller DS, Farhi A, Pinkerton N et al (2000) Activating mineralocorticoid receptor mutation in hypertension exacerbated by pregnancy. Science 289:119-123

12. Gupta-Malhotra M, Banker A, Shete $S$ et al (2015) Essential hypertension vs. secondary hypertension among children. Am JHypertens 28:73-80

13. Hansson JH, Nelson-Williams C, Suzuki H et al (1995) Hypertension caused by a truncated epithelial sodium channel gamma subunit: genetic heterogeneity of Liddle syndrome. Nat Genet 11:76-82
14. Jackson RV, Lafferty A, Torpy DJ et al (2002) New genetic insights in familial hyperaldosteronism. Ann NY Acad Sci 970:77-88

15. Jonsson JR, Klemm SA, Tunny TJ et al (1995) A new genetic test for familial hyperaldosteronism type $\mathrm{I}$ aids in the detection of curable hypertension. Biochem Biophys Res Commun 207:565-571

16. Liddle GW, Bledsoe T, Coppage WS (1963) A familial renal disorder simulating primary aldosteronism but with negligible aldosterone secretion. Trans Assoc Am Phys 76:199-231

17. Lifton RP, Dluhy RG, Powers M et al (1992) Hereditary hypertension caused by chimaeric gene duplications and ectopic expression of aldosterone synthase. Nat Genet 2:66-74

18. Litchfield WR, Anderson BF, Weiss RJ et al (1998) Intracranial aneurysm and hemorrhagic stroke in glucocorticoid-remediable aldosteronism. Hypertension 31:445-450

19. Mansfield TA, Simon DB, Farfel Z et al (1997) Multilocus linkage of familial hyperkalaemia and hypertension, pseudohypoaldosteronism type II, to chromosomes 1q31-42 and 17p11-q21. Nat Genet 16:202-205

20. Morineau G, Sulmont V, Salomon R et al (2006) Apparent mineralocorticoid excess: report of six new cases and extensive personal experience. J Am Soc Nephrol 17:3176-3184

21. Mulatero P, Monticone S, Rainey WE et al (2013) Role of KCNJ5 in familial and sporadic primary aldosteronism. Nature reviews. Endocrinology 9:104-112

22. Ncbi ClinVar https://www.ncbi.nlm.nih.gov/ clinvar/.Zugegriffen: 14. Juli 2018

23. Neuhauser H, Kuhnert R, Born S (2017) 12-MonatsPrävalenz von Bluthochdruck in Deutschland. JHealth Monit 2(1):57-63

24. Nimkarn S, Gangishetti PK, Yau M et al (1993) 21-Hydroxylase-deficient congenital adrenal hyperplasia. In: Adam MP, Ardinger $\mathrm{HH}$, Pagon RA, Wallace SE, Bean LJH, Stephens K, Amemiya A (Hrsg) GeneReviews((R)). University of Washington, Seattle, Seattle

25. Pinggera A, Mackenroth L, Rump A et al (2017) New gain-of-function mutation shows CACNA1D as recurrently mutated gene in autism spectrum disorders and epilepsy. Hum Mol Genet 26:2923-2932

26. Rimoldi SF, Scherrer U, Messerli FH (2014) Secondary arterial hypertension: when, who, and how to screen? Eur Heart J 35:1245-1254

27. Salih M, Gautschi I, Van Bemmelen MX et al (2017) A missense mutation in the extracellular domain of alphaEnaC causes Liddle syndrome. J Am Soc Nephrol 28:3291-3299

28. Scholl UI, Goh G, Stolting G et al (2013) Somatic and germline CACNA1D calcium channel mutations in aldosterone-producing adenomas and primary aldosteronism. Nat Genet 45:1050-1054

29. Scholl UI, Stolting G, Nelson-Williams $C$ et al (2015) Recurrent gain of function mutation in calcium channel CACNA1H causes early-onset hypertension with primary aldosteronism. eLife 4:e6315

30. Scholl UI, Stolting G, Schewe J et al (2018) CLCN2 chloride channel mutations in familial hyperaldosteronism type II. Nat Genet 50:349-354

31. Shibata S, Zhang J, Puthumana J et al (2013) Kelchlike 3 and Cullin 3 regulate electrolyte homeostasis via ubiquitination and degradation of WNK4. Proc Natl Acad Sci USA 110:7838-7843

32. Shimkets RA, Warnock DG, Bositis CM et al (1994) Liddle's syndrome: heritable human hypertension caused by mutations in the beta subunit of the epithelial sodium channel. Cell 79:407-414

33. Stowasser M, Gordon RD, Tunny TJ et al (1992) Familial hyperaldosteronism type II: five families with a new variety of primary aldosteronism. Clin Exp Pharmacol Physiol 19:319-322

34. Warren HR, Evangelou E, Cabrera CP et al (2017) Genome-wide association analysis identifies novel blood pressure loci and offers biological insights into cardiovascular risk. Nat Genet 49:403-415

35. Yang CL, Angell J, Mitchell R et al (2003) WNK kinases regulate thiazide-sensitive $\mathrm{Na}-\mathrm{Cl}$ cotransport. JClin Invest 111:1039-1045 